\title{
An agent-based simulation of extirpation of Ceratitis capitata applied to invasions in California
}

\author{
Nicholas C. Manoukis • Kevin Hoffman
}

Received: 29 January 2013/ Accepted: 19 June 2013/Published online: 29 June 2013

(C) The Author(s) 2013. This article is published with open access at Springerlink.com

\begin{abstract}
We present an agent-based simulation (ABS) of Ceratitis capitata ("Medfly") developed for estimating the time to extirpation of this pest in areas where quarantines and eradication treatments were immediately imposed. We use the ABS, implemented in the program MED-FOES, to study seven different outbreaks that occurred in Southern California from 2008 to 2010. Results are compared with the length of intervention and quarantine imposed by the State, based on a linear developmental model (thermal unit accumulation, or "degree-day"). MED-FOES is a useful tool for invasive species managers as it incorporates more information from the known biology of the Medfly, and includes the important feature of being demographically explicit, providing significant improvements over simple degree-day calculations. While there was general agreement between the length of quarantine by degree-day and the time to extirpation indicated by MED-FOES, the ABS suggests that the margin of safety varies among cases and that in two cases the quarantine may have been excessively long. We also examined changes in the number of individuals over time in MED-FOES and conducted a sensitivity analysis for one of the outbreaks to explore the role of various input parameters on simulation outcomes. While
\end{abstract}

Communicated by R. Meyhöfer.

N. C. Manoukis $(\bowtie)$

US Pacific Basin Agricultural Research Center, United States Department of Agriculture-Agricultural Research Service, Hilo, HI, USA

e-mail: nicholas.manoukis@ars.usda.gov

K. Hoffman

California Department of Food and Agriculture, Sacramento, CA, USA

e-mail: khoffman@cdfa.ca.gov our implementation of the ABS in this work is motivated by $C$. capitata and takes extirpation as a postulate, the simulation is very flexible and can be used to study a variety of questions on the invasion biology of pest insects and methods proposed to manage or eradicate such species.

Keywords Simulation - Model · Medfly - Quarantine . Agent-based modeling

\section{Introduction}

Ceratitis capitata (Mediterranean fruit fly, "Medfly") is a major threat to agriculture around the world because it can infest a large variety of commercial fruit crops (Liquido et al. 1990) and is able to persist in a wide variety of habitats (Messenger 1959). Growing regions that do not have resident populations of this species established or that are part of "Medfly-free" zones may occasionally experience incursions of this species, usually detected by finds of immature stages in fruit or by adult individuals being captured in traps under intensive surveillance programs. When C. capitata is found in an area where it is not known to be established, there are usually quarantine, eradication, and phytosanitary measures implemented to eliminate the Medfly from the infested area (CDFA 2012). Treatments are planned to last only long enough to eliminate this insect and then are lifted to allow the movement of produce safely to market without quarantine or the undue costs of excessive treatments.

One major problem that arises when commodity quarantines and other control measures are imposed as the result of a find (or "outbreak") in a C. capitata "free" area is to determine how long to maintain quarantine and eradication measures before they can be lifted. This paper 
describes an agent-based simulation (ABS) developed to estimate how long a small population of Medfly is likely to persist in a quarantine area post-detection while the area is managed under quarantine security and eradication procedures. Specifically, we use an ABS implementation called MED-Fly Outbreak and Eradication Simulation (MEDFOES) to study the time to extirpation (local extinction) of C. capitata following seven actual detections of this pest that occurred in Southern California between 2008 and 2010. This is done through detailed modeling of individual flies and their life expectations based on probability density functions of survival, developmental rate, and reproductive potential determined from the actual population studies.

The state of California maintains an active surveillance network of traps, as well as a preventative release program in the Los Angeles basin using the sterile insect technique (SIT) (Dowell et al. 2000). These programs are maintained because potential establishment of the Medfly could be expected to cause approximately 1.2 billion dollars in costs to the state economy in the first year following the determination of establishment (Siebert and Cooper 1995). In addition to SIT control methods, the eradication teams implement fruit stripping, apply bait sprays, and massively increase trapping in attempts to kill all the flies in the area (Gilbert et al. 2010). Despite these efforts, wild C. capitata are found in the state one or more times per year, in a seasonal pattern concentrated in the summer and fall (Carey 1991). Medfly is also periodically found in other US Mainland states such as Florida (Simberloff et al. 1997), although not as frequently as observed in Southern California.

Currently, commodity quarantines and treatment duration in California following a numerically significant Medfly find are established and maintained based on a thermal unit accumulation calculation ("degree-days"; Gilbert et al. 2010), which is the prescribed method to estimate the developmental time of the insect given ambient temperatures from the infestation location. The typical quarantine and treatment duration is calculated to be the time required for three generations of the insect to pass ("three complete life cycles") under local temperatures.

The first detection of Medfly in California occurred in 1975 , and it has reoccurred regularly since the early 1980 s (Carey 1996). It has been argued that Medfly may actually be established and persist at a low population density in California, with numbers increasing periodically to just above the detection level (Carey 1991). However, this matter remains unresolved, as others have argued that the Medfly in California is an example of a "metainvasion," consisting of multiple sequential or overlapping introductions (Davies et al. 1999). Still other researchers have maintained that Medfly is repeatedly eradicated from the state (Haymer et al. 1997) or that different situations arise in different regions (Bonizzoni et al. 2001; Gasperi et al. 2002), making the situation more complex across the entire state of California.

The question of whether Medfly is in fact established in California is beyond the scope of this paper; our ABS assumes extirpation as a precept, which in the case of Medfly in Southern California may mean localized extinction from a relatively small area. This local extinction is expected to occur under quarantine and eradication procedures as implemented by the California Department of Food and Agriculture (CDFA) and the US Department of Agriculture (CDFA 2012). Applying ABS models to this situation allows an independent assessment of the extirpation process and in addition to being a practical application of a simulation modeling approach, it is a novel application of an individual-level model. The ABS presented here can (1) be considered as an independent assessment of the current three generation degree-day calculation and (2) can be used as a method of analysis to show ways in which quarantine length might be adjusted to increase effectiveness, reduce costs, or ensure uniform likelihood of extirpation following Medfly finds in a wide area.

Computer simulations of complex systems in biology are increasingly common, driven by rapid increases in processing speed, decreases in processing cost and complexity, and the realization that simulations can aid the researcher and program manager in considering added complexity of the real world, while freeing them from limitations imposed by analytical solutions of complex mathematical processes (Huston et al. 1988). The latter is particularly true for ABS models (also called "IndividualBased," or "Multi-Agent" models), which can be minimally defined as simulations where individuals are described as unique and autonomous, and where they may interact with each other and their environment on a local level (Railsback and Grimm 2012). Such simulations describe a system of interest "from the bottom up." This is accomplished by implementing the model using many replicate constituent agents or entities (insects in this case) and then observing the system behaviors and dynamics that result from those entities interacting autonomously with their environment, and often with each other as they would in the real world (Bonabeau 2002). Such an approach is particularly effective when working with small populations of entities as random events can significantly affect such populations. In the present study individual $C$. capitata adults and immatures are instantiated as agents (or entities) in the simulations of pest outbreaks and allowed to live, reproduce, and die based on their interactions with realistic environmental conditions. 
While the freedom to include any degree of complexity in an ABS comes at a cost (DeAngelis et al. 1994; Grimm 1999; Hales et al. 2003), the pragmatic and paradigmatic benefits of the Agent-Based approach can be significant for many different areas of biology, including those concerned with pest invasions (Macal and North 2010; Railsback and Grimm 2012). These benefits have infrequently been applied to the area of insect invasion and pest management [Vinatier et al. 2011, but see Crespo-Pérez et al. (2011) for a recent example]. An ABS can be a natural description (Fry et al. 1989) of the invasion biology of an introduced insect such as the Medfly. It is particularly useful that an ABS can include discontinuities, thresholds, large effects of stochasticity in small population, and any degree of heterogeneity required to make the simulation realistic and useful for solving practical problems. In the case of the Medfly, it enables simulation of the complete life cycle of each individual (Railsback and Grimm 2012) insect in the population, which is of central importance to the question of quarantine length in our Medfly eradication assessment. Thus, the ABS approach is extremely flexible and can be modified or expanded with more data-derived information as required to address a particular problem or to ask different questions about insect invasions and their environmental repercussions. Finally, our ABS is numerically explicit and stochastic, which means that it can produce predicted expectations and associated variance, making these calculations statistically realistic and robust for realworld problem solving.

\section{Materials and methods}

The Medfly ABS is defined using the "Overview, Design concepts, and Details" (ODD) protocol (Grimm et al. 2006, 2010), as a standardized method for describing individualbased models. The computer program we developed using ABS procedures, "MED-FOES," is available for free as a compiled binary with the source code and documentation, online (see http://ars.usda.gov/pwa/hilo/software or http:// medfoes.sourceforge.net).

\section{Purpose}

The purpose of MED-FOES as presented here is to estimate the time to extirpation of invasive $C$. capitata under California conditions or other related habitats where Medfly-free areas might have similar climatic patterns. Results from executions of the simulation are based on parameters and input data from real outbreaks which have been used to evaluate the length of quarantine and treatment following previous and future finds of $C$. capitata in particular exclusion areas.
Entities, state variables, and scales

The basic entity (or agent) in our ABS is an individual insect or Medfly. These individuals are created, develop, reproduce, and die throughout the simulation in similar patterns that they would be expected to follow in nature. Figure 1 is a schematic representation of the life cycle of a single agent in the simulation, showing the stages or states in which the simulated insect can occur. During a simulation time step of $1 \mathrm{~h}$, each individual insect has the potential to transition stages (develop and molt), die from natural causes, or die from human-induced mortality, in that order. If an individual dies it is removed from the simulation and no longer affects the overall calculations in the model. To further reduce the complexity and number of individual calculations, only female flies are simulated as they are the ones important in the reproduction and infestation cycle of interest to the quarantine managers.

Individual simulated insects are characterized by state variables indicating their age (in hours), developmental stage, sexual maturity, development parameters (the thermal constant $K$ and base developmental temperature $T_{\min }$ for each stage), and a development time modifier. The individual insects (agents) are referenced to the overall "Population," which tracks population-level data such as cumulative numbers of births and deaths. The ambient temperature for the current time step is also tracked through time, as are specific parameters relevant to each individual insect. Table 1 provides an overall summary of all the input parameters used throughout the model.

Process overview and scheduling

As mentioned previously, our ABS functions using a 1-h time step. This level of detail is important as we consider

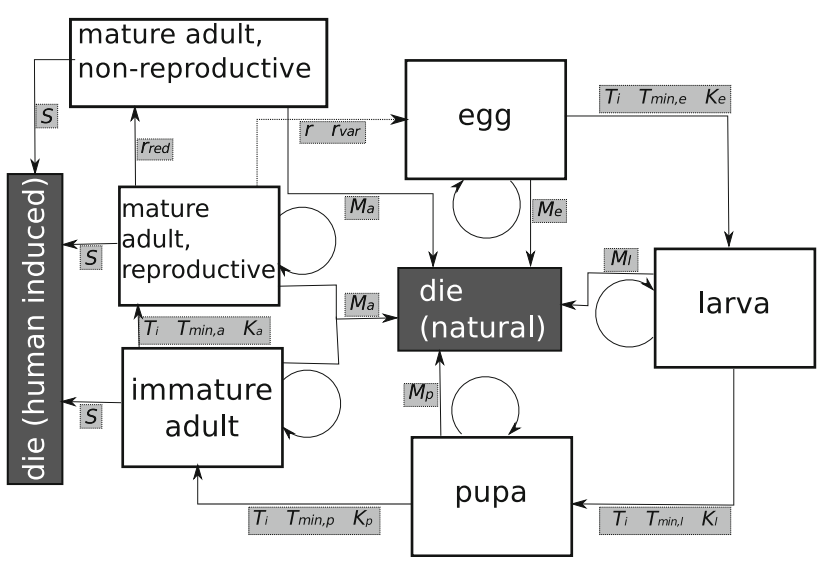

Fig. 1 Schematic representing states and transitions possible for insects in the ABS under the simplest mode (uniform transition probability and temperature-independent mortality). The individuallevel parameter $\gamma$ is omitted for clarity 
Table 1 Input parameters of the ABS described in this paper

\begin{tabular}{llll}
\hline Input parameter & Symbol & Units/type & Description \\
\hline Temperature & $T_{i}$ & ${ }^{\circ} \mathrm{C}$ & Hourly temperature for time $i$ \\
Initial population size & $N_{0}$ & Insects & Number of adult females at start of simulation \\
Daily mortality & $M_{x}$ & Proportion & Estimated daily mortality by stage \\
Base temperature & $T_{\mathrm{min}, x}$ & ${ }^{\circ} \mathrm{C}$ & Temperature at which development rate is zero \\
Thermal constant & $K_{x}$ & Days & No. of day-degrees above $T_{\text {min }}$ for stage transition \\
Maximum temperature & $T_{\max }$ & ${ }^{\circ} \mathrm{C}$ & Development halts above this temperature \\
Developmental model & $D_{\mathrm{m}}$ & Flag & Developmental transition model to use \\
Variation in development rate & $\gamma$ & Proportion & SD of mean thermal units needed for stage transition \\
Reproductive output & $r$ & Eggs & Number of eggs produced by female per reproduction \\
Reproductive variance & $r_{\mathrm{var}}$ & Eggs & Variance of $r$ \\
Sterilization rate & $r_{\mathrm{red}}$ & Proportion & Reduction in $r$ per day \\
Human-induced mortality & $S$ & Proportion & Daily mortality from countermeasures on adults \\
Time to countermeasures & $t_{\mathrm{S}}$ & days & Time from the first find to start human intervention \\
Maximum number of flies & $N_{\mathrm{m}}$ & Insects & Maximum number of insects allowed in simulation \\
\hline
\end{tabular}

All but $T_{i}, t_{\mathrm{S}}$, and $T_{\max }$ might be varied for the analysis of a given outbreak scenario. $D_{\mathrm{m}}$ and $N_{\mathrm{m}}$ are related to modeling and computation decisions, and are not varied between simulations of a given outbreak. $x=\mathrm{e}$ (eggs), 1 (larvae), $\mathrm{p}$ (pupae), or a (adults)

this high resolution beneficial for overall accuracy, especially when a handful of very low or very high temperatures can have a large effect on the simulation outcome. Since hourly temperature data are available from the field, and detailed effects might be lost if daily averages were used, the simulation works to incorporate Medfly biology and development at this timescale. Thus, hourly time steps are executed in the simulation from initiation until all agents are predicted to be dead, or the maximum number of allowed steps (determined by the number of hourly temperatures available in the input file) has been met.

One hour has passed in the simulation when all the flies have been given the opportunity to age one developmental step. The following events are considered sequentially for each fly to age one step:

(1) Determine if a stage transition will occur. Occurrence of a stage transition can be modeled either as a random event with the probability related only to the temperature at the current hour or as a more biologically realistic accumulation of "thermal units" with stochastic variability between individual insects. The model increments the stage and changes the developmental parameters of the individual insect if stage transition occurs to the individual entity within that time step.

(2) If the insect is a mature adult and the intervention/ quarantine period has not begun, one time per day the model simulates the fly laying eggs. The number of eggs produced has a mean and variance based on realistic fecundity patterns from field and laboratory data. After the start of intervention, a portion of the mature adults may become sterile each day, simulating the SIT releases. Offspring generated after intervention may optionally be sterile or subject to the same reduction in fertility as their parents.

(3) Determine if the fly dies from natural causes. This is accomplished in one of two ways: (i) Natural death is a random event that occurs with a set stage-specific probability for a given simulation or (ii) stage- and temperature-specific mortality is calculated for each hour of the simulation (see "Submodels"). To enhance the realism, mode (ii) was used in all simulations applied to this study.

(4) If the insect is an adult and the intervention period has begun, the model then determines if the fly dies from the intervention (human-induced death). Humaninduced death is a random event that occurs with a set probability for a given simulation.

\section{Design concepts}

\section{Emergence}

Individual-level variation in immature developmental rates and adult emergence, mortality, and reproductive output combined with stochastic responses to temperature lead to emergent population-level behaviors in the model. Although the Medfly population is inclined to increase based on its inherent reproductive capacity represented in the model, the initiation of countermeasures $\left(t_{\mathrm{S}}\right)$ imposed by the restriction on reproduction by fruit stripping, SITinduced reduction in fecundity and human-induced mortality imposed by bait spraying and massively increased trapping cause a general population decline as seen in the 
real world. It is important to note that any eggs laid after initiation of countermeasures (at $t_{\mathrm{S}}$ ) are considered sterile in the simulations, reflecting the expected lack of host fruit in the quarantine area following activation of countermeasures. This was implemented in the MED-FOES model to more realistically mimic the exact situation in the field; however, the generalized implementation of this ABS model allows for fertile offspring to be simulated if desired.

\section{Stochasticity}

At the agent level, stochasticity occurs primarily in association with developmental times, reproduction levels, and mortality rates. For developmental time, there is an individual variation in each individual's response to ambient temperature. Reproductive output varies between adult females, with some flies being more fecund than others on an individual level. For mortality, a probability of death is calculated (see "Mortality" in Submodels section below) but the actual occurrence of death for a given individual is based on a pseudo-random number drawn each hour, and thus rates are determined both deterministically based on observed response function data and then modified stochastically to provide statistical realism in the actual mortality response within the simulation.

\section{Observation}

Two types of output result from a single execution of the MED-FOES model. The first is a summary file containing aggregate information like the total number of time steps (hours) that the simulation has completed, the number of alive insects throughout the simulation, and cumulative numbers of eggs produced and the deaths of adult flies. This summary also includes critical model run information (parameters, execution date, time, etc.). The second file is a detailed view of the simulation run that includes ambient temperature (min, max, and mean) and the number of living agents in each developmental stage on a daily basis. This more detailed file provides information on Medfly cohorts, especially immatures that may be incorporated in the larger habitat but unavailable to assess through the standard trapping practices.

\section{Initialization}

In order to initiate the model, we require reasonable starting population sizes that are derived from two pieces of information based on data available in the literature and/ or the monitored field situation: (1) an estimate of the size of the adult female population and (2) an estimate of the age structure of the population (especially eggs and larvae) that cannot be assessed through adult trapping. With these two estimated pieces of information, we can project the number of individuals in each life stage of the invasive population of Medfly and then initiate the model in its operational mode.

From field monitoring data provided by the California Department of Food and Agriculture (CDFA) on each of the simulated outbreaks, we have the number of flies that were found at the time of first detection. We consider flies caught in the first 3 days as the number caught at first detection. We use these values as an instantaneous measure of the adult population within the area of concern. We also know what the trapping grid was like at the time of the finds. For example, in 2008-2010: 5 Jackson traps with Trimedlure and 5 McPhail traps with protein bait were deployed per square mile (Gilbert et al. 2010). Based on release-recapture work conducted in Hawaii, it has been estimated that the attractive ability of such traps is estimated to be $0.04 \%$ of the population to one trap per square mile per adult life span (Steiner 1967, 1969). Steiner (1967) notes, however, that he suspected movement of the flies out of the study area which would somewhat alter the assessment values. More recent works by Cunningham and Couey (1986) and Lance and Gates (1994) indicate a somewhat better sensitivity. At five traps per square mile, which is the standard density in urban parts of Southern California, the former estimates sensitivity to be $2.21 \%$ of males per adult life span. Lance and Gates (1994) found a lower rate, around $0.6 \%$.

A reasonable estimate for the detection efficiency of Trimed lure is probably around $1.0-1.5 \%$. However, we must also consider the additional detection ability afforded by the McPhail traps. The available literature suggests that protein lures are approximately as effective as Trimed lure in attracting Medfly (Grout et al. 2011; Katsoyannos et al. 1999; Midgarden et al. 2004). For Medfly in our analysis, then, we assume a $2-3 \%$ sensitivity of the trapping grid. Given a number of adult females derived from assuming an even sex ratio, we use the projected stable age structures from Vargas et al. (1997) at $24{ }^{\circ} \mathrm{C}$ to infer the number of individuals in immature stages that should exist in the population at large, even though we have no actual measurement of their densities. To be conservative, we ran a second set of simulations with the initial age distribution from Vargas et al. (2000) and found very similar results, thus the model does not seem to be particularly sensitive to these initial age distribution parameters. 
Input data

\section{Hourly temperatures}

MED-FOES simulations use hourly temperature data acquired from the California Irrigation Management Information System (CIMIS; data available online at http:// wwwcimis.water.ca.gov), which maintains weather stations logging at this rate all across the state of California. These data are available for many monitoring sites via the internet and are accuracy checked for all locations. In cases where data were missing from the time periods and locations used, they were estimated from daily min-max averages from the previous 5 years using the method described below in the "Submodels" section.

\section{Outbreak events}

We have simulated seven outbreak events that occurred in Southern California from 2008 to 2010. The locations of the seven outbreaks and additional details are provided in Table 2. The state of California imposes quarantine on fruit entering or leaving an area of $\sim 210 \mathrm{~km}^{2}$ around each find of Medfly according to a published set of rules (CDFA 2012). The standard length of each quarantine is then determined using a fixed degree-day developmental transition model (Carey 1992) based on temperatures for the location of the find. The number of degree-days is calculated to allow the time needed for three generations of Medfly to pass (CDFA 2012). This length of time has historically proven effective in avoiding recurrence of Medfly detection in an area after interventions and when completed, the quarantine and other interventions are terminated. Trapping continues in place to both assess effectiveness and monitor for subsequent reinvasions and/ or population increases.

Table 2 Dates and locations of outbreaks analyzed with ABS plus the distance to the nearest CIMIS weather station

\begin{tabular}{llllrr}
\hline Outbreak & Approx address & Find & Q. end & WS & dist \\
\hline Santa Monica & Warwick Ave. & $10 / 28 / 09$ & $08 / 29 / 10$ & 99 & 2.4 \\
Fallbrook & Punta de Lomas & $10 / 29 / 09$ & $07 / 16 / 10$ & 62 & 22.2 \\
Spring Valley & Leland St. & $02 / 05 / 09$ & $08 / 27 / 09$ & 147 & 16.7 \\
Imperial beach & 11th St. & $07 / 30 / 09$ & $12 / 21 / 09$ & 184 & 16.3 \\
Mira Mesa & Flanders Dr. & $05 / 20 / 09$ & $11 / 03 / 09$ & 150 & 2.1 \\
Escondido & N Rose St. & $09 / 09 / 09$ & $07 / 26 / 10$ & 153 & 10.0 \\
Cajon & Lisa Terrace. & $11 / 07 / 08$ & $07 / 15 / 09$ & 184 & 22.6 \\
\hline
\end{tabular}

Dates are given in MM/DD/YY format

$Q$ quarantine, WS ID number of the nearest CIMIS weather station, dist distance from CIMIS weather station to outbreak location, in kilometers
Submodels

\section{Development}

Many reports on the development of pest or other insects in the literature give the mean time to transition in days or hours $(d)$ for each stage as affected by a range of constant fixed temperatures $(T)$. These are commonly obtained via linear regression of the developmental rate against fixed temperatures $(1 / d=a+b T)$ (Campbell et al. 1974), which gives a clear linear relationship for temperatures between about 16 and $30{ }^{\circ} \mathrm{C}$ for Medfly (Grout and Stoltz 2007). The commonly reported base developmental temperature $T_{\min }$ and developmental constant $K$ (which is the time-to-transition) are related to the terms of the linear regression model above, $T_{\min }=-a / b$ and $K=1 / b$.

We implemented a developmental model based on the thermal unit accumulation approach (Fletcher 1989), where each degree above $T_{\min }$ for each hour counts toward a required threshold $C$ (measured in the laboratory) for stage transition. We add variation to each individual fly when it is instantiated in the form of a variable $\gamma$, which is the standard deviation of the development time as a proportion of the development time for each stage. Thus, when

$C+\gamma \leq \sum_{t=0}^{i} T_{i}-T_{\min }$

from the time of insect creation (0) to the current time $i$, stage transition occurs. Note that the value of $C$ is stagespecific, and constant across individuals.

\section{Reproduction}

Every $24 \mathrm{~h}$ prior to quarantine and control intervention, every adult Medfly is simulated to lay eggs. In this sense, the model only considers females. The mean number of eggs and variance in reproductive output are set by the variables $r$ and $r_{\mathrm{var}}$. After human intervention is initiated reproduction is curtailed at a set daily rate, which is denoted as $r_{\text {red. }}$. This variable was included based on previous models of the SIT and its effects on target insect populations (Knipling 1979), simulating the fact that, over time wild females mating with sterile males keeps them from producing viable offspring. In the MED-FOES model, each mature adult fly that exists after $t_{\mathrm{S}}$ is subject to an additional loss of reproductive ability, each day with probability $r_{\text {red }}$.

\section{Mortality}

Two methods of simulating natural mortality are included in our ABS implementation of MED-FOES. The simplest 
approach is to set a fixed stage-specific daily mortality rate, which we denote as $M_{x}$. Each hour of the simulation, and for each insect at stage $x$, a random double precision floating point number between 0 and 1 is drawn and if it is lower than $M_{x}^{1 / 24}$ then the insect dies.

In the case of Medfly there are reliable data available on the effect of temperature on daily mortality rates, so we used the second method for simulating death. We used the stagespecific quadratic relationships from Gutierrez and Ponti (2011) and then varied the degree of mortality between runs by varying the mortality at the optimum temperature (20-25 ${ }^{\circ} \mathrm{C}$ ). When used this way, we denote the stage-specific mortality as $M_{x}^{*}$, indicating that this is the mortality at optimum, and then it is subsequently affected by temperature.

Table 3 provides the range of fixed mortality rates for each stage of the Medfly life history at approximately the optimum temperatures of $20-25^{\circ} \mathrm{C}$ as determined by our survey of the literature. We added temperature-dependent mortality ( $\mu_{x}$, where $x$ may be e, eggs; 1 , larvae; $\mathrm{p}$, pupae or a, adults) with parameters from (Gutierrez and Ponti 2011). The equations used were

$$
\begin{gathered}
\mu_{\mathrm{e}, 1}=0.00040 T^{2}-0.0145 T+0.1314 \\
\mu_{\mathrm{p}}=0.00050 T^{2}-0.0207 T+0.2142 \\
\mu_{\mathrm{a}}=0.00049 T^{2}-0.0187 T+0.1846
\end{gathered}
$$

Additional mortality of adult flies at a rate $S$ occurs after human intervention at time $t_{\mathrm{S}}$. This represents mortality from human activities such as spraying with insecticides, applying bait sprays, and increasing trapping to help control the outbreak. We set the effect of human countermeasures to vary between relatively ineffective to medium efficiency: 0.5-5.0 \% and applied it as additional fly deaths per day. We do not include additional mortality in stages prior to adult as there were no measures specifically targeted against these stages in California at the time of the outbreaks under study, except for fruit stripping $100 \mathrm{~m}$ around the location of a find.

After $t_{\mathrm{S}}$, any new flies produced may survive, but they themselves will not reproduce due to the effects of the SIT and pesticide application programs. Flies that were alive before $t_{\mathrm{S}}$ may still reproduce, though they are subject to lose that ability at a rate set by $r_{\text {red }}$ (see above). These modeling decisions represent a conservative middle ground between assuming that fruit stripping is $100 \%$ effective with no more reproduction by any flies after countermeasures are initiated, and the perspective that the population can freely reproduce and thus would never be extirpated.

\section{Estimating hourly temperatures}

When hourly temperature values are not available by direct measurement, they can be estimated. In our analysis we
Table 3 Estimates of simulation parameter ranges based on literature review

\begin{tabular}{lcl}
\hline Input parameter & Range (min-max) & References \\
\hline$N_{0}^{\mathrm{a}}$ & $33-100$ & $1-4$ \\
$M_{\mathrm{e}}^{*}$ & $0.0198-0.1200$ & $5-11$ \\
$M_{\mathrm{l}}^{*}$ & $0.0068-0.0946$ & $5-10$ \\
$M_{\mathrm{p}}^{*}$ & $0.0016-0.0465$ & $5-10,19$ \\
$M_{\mathrm{a}}^{*}$ & $0.0245-0.1340$ & $7,12,13,20,21$ \\
$S$ & $0.005-0.050$ & $\mathrm{~N} / \mathrm{A}$ \\
$T_{\min , \mathrm{e}}$ & $9.6-12.5$ & $5-8,11$ \\
$K_{\mathrm{e}}$ & $27.27-33.80$ & $5-8$ \\
$T_{\min , 1}$ & $5.0-10.8$ & $5-8$ \\
$K_{\mathrm{l}}$ & $94.50-186.78$ & $5-8$ \\
$T_{\min , \mathrm{p}}$ & $9.1-13.8$ & $5-7$ \\
$K_{\mathrm{p}}$ & $123.96-169.49$ & $5-7$ \\
$T_{\min , \mathrm{a}}$ & $7.9-9.9$ & $5,6,13$ \\
$K_{\mathrm{a}}$ & $58.20-105.71$ & $5,6,13$ \\
$r$ & $5.0-35.0^{\mathrm{b}}$ & $1,2,7,13,14-18$ \\
$r_{\text {red }}$ & $0.5-1.0$ & 22,23 \\
\hline
\end{tabular}

Age structure (proportions) was based on Vargas et al. 1997: 0.436 (e, eggs), 0.403 (l, larvae), 0.137 (p, pupae), 0.012 [a, adults (immature)], 0.012 [(a, adults (mature)]. $\gamma$ was set to 0.05 and $T_{\max }$ to 35 for all simulations

1 Steiner 1967, 2 Steiner 1969, 3 Cunningham and Couey 1986, 4 Lance and Gates 1994, 5 Duyck and Quilici 2002, 6 Grout and Stoltz 2007, 7 Shoukry and Hafez 1979, 8 Vargas et al. 1996, 9 Papadopoulos et al. 2002, 10 Papachristos et al. 2008, 11 Messenger and Flitters 1958, 12 Rivnay 1950, 13 Vargas et al. 1997, 14 Back and Pemberton 1918, 15 Christenson and Foote 1960, 16 Rossler 1975, 17 Kaspi et al. 2002, 18 Harris et al. 1991, 19 El Keroumi et al. 2010, 20 Diamantidis et al. 2009, 21 Carey 2011, 22 Knipling 1979, 23 Sawyer et al. 1987

${ }^{\text {a }}$ Number of adult females per adult fly found

b $r_{\text {var }}$ estimated at 3.57 based on sample of $r$ values from the literature

were able to obtain direct measurements for almost all the hours being simulated (see below), but when data were missing we used the method described by Campbell and Norman (1997) to obtain estimates. The method involves using two terms of a Fourier series fitted to a longer term hourly average:

$\Gamma(t)=0.44-0.46 \sin (\omega t+0.9)+0.11 \sin (2 \omega t+0.9)$

where $\omega=/ 12$, and $\mathrm{t}$ is time of day in hours, with $t=12$ at solar noon. The temperature for any time of a day $i$ can be estimated as follows:

$T(t)= \begin{cases}T_{x, i-1} \Gamma(t)+T_{n, i}[1-\Gamma(t)] & 0<t \leq 5 \\ T_{x, i} \Gamma(t)+T_{n, i}[1-\Gamma(t)] & 5<t \leq 14 \\ T_{x, i} \Gamma(t)+T_{n, i+1}[1-\Gamma(t)] & 14<t<24\end{cases}$

where $T_{x}$ is the daily maximum temperature and $T_{n}$ is the daily minimum. 


\section{Validation}

Agent-based methods can be powerful for simulating complex systems, but require careful testing and validation through comparison against real-world data (Bonabeau 2002). To validate the behavior of a population of flies using ABS we compared MED-FOES output under simplified conditions of constant temperature, reproduction, and mortality to that from a standard Leslie matrix projection (Leslie 1945; Lewis 1942). This is a well-studied method, and we made these comparisons using actual Medfly data and comparisons with other published models. Carey (1982) utilized the reproductive, survivorship, and developmental data from Shoukry and Hafez (1979) to study Medfly demography using a Leslie matrix model. We used the same parameters as they applied to MED-FOES with the same data and compared the results, so in the current context validation is defined as testing the ABS against a known method to assess its behavior under simple conditions.

\section{Results}

\section{Validation}

Figure 2a shows a 20-day iteration of the Leslie matrix projection of Carey (1982) and Fig. 2b shows the same length of time simulated with the MED-FOES model. In both cases the population is initiated with 100 sexually mature females, allowing direct comparison. The results of the ABS are quite similar to those from the Leslie matrix approach. We note that in the case of these parameters the population size is decreasing through time under both methods, so there is increasing variance in the later days of the simulation. This is not seen in the Leslie matrix projection results (Fig. 2a) because that method is deterministic and not affected by small insect number as would be expected in the real world as densities decrease. It is noteworthy that the overall number of flies drops over time primarily as the daily survivorship of larvae derived by Carey (1982) is very low at 0.6 , thus very few insects can transition to mature adults to reproduce.

The behavior of populations within the ABS is determined by events occurring at the level of individual agents, subject to stochastic effects. Figure 3 shows the relationship between mean daily temperature and the proportion of agents in all stages that died during the same day for a sample of 100 executions of MED-FOES from the analysis of the outbreak in Fallbrook. It is clear that there is some relationship between the observed mortality and the expected level of adult mortality $\left(M_{\mathrm{a}}\right)$, indicated by the gray lines. However, clearly there are also data points
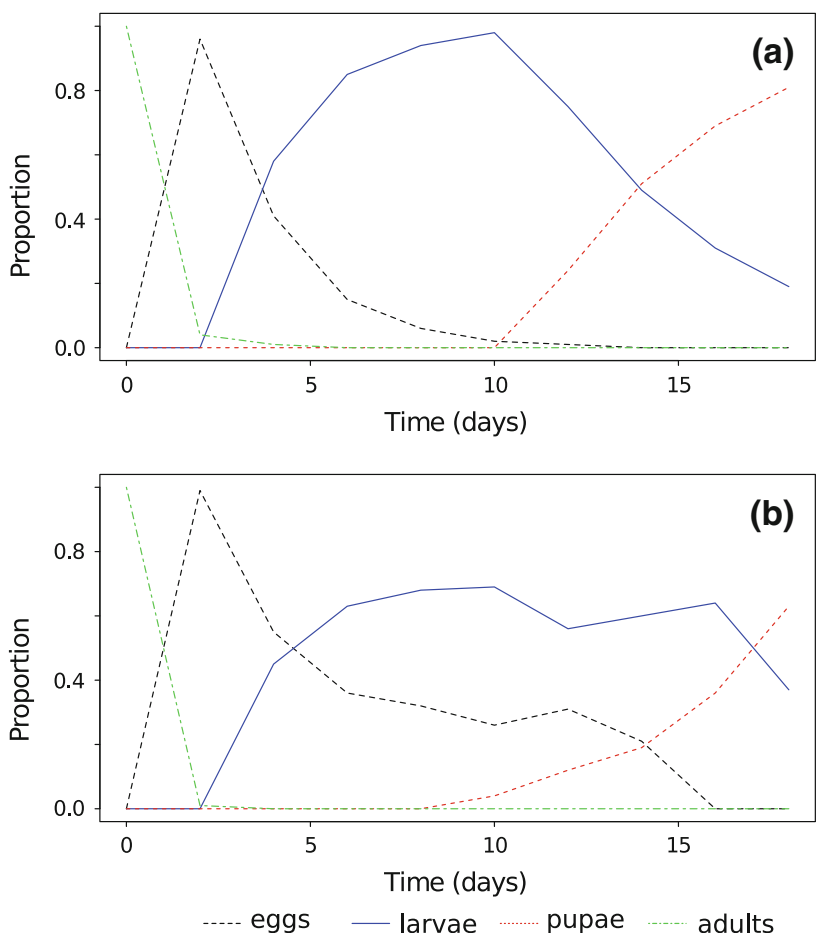

Fig. 2 Proportion of insects in each developmental stage over 20 days under two modeling methods. a Leslie matrix projection and $\mathbf{b}$ ABS. Both methods used parameters measured in real insects (Shoukry and Hafez 1979), and exhibited similar demographic trends (decreasing number over time). Parameters for the Leslie matrix model were as shown in Carey (1982), with 40 age classes and the initial number of mature females $N_{30,0}=100$. For the ABS developmental parameters were taken directly from Shoukry and Hafez (1979): $T_{\text {min,e }}=9.6, \quad T_{\min , 1}=5.0, \quad T_{\min , \mathrm{p}}=13.8, \quad K_{\mathrm{e}}=33.4$, $K_{1}=186.8, K_{\mathrm{a}}=124.0$. Mortality rates per stage were fixed to match those used by Carey (1982): $M_{\mathrm{e}}=0.00, \quad M_{1}=0.60$, $M_{\mathrm{p}}=0.20, M_{\mathrm{a}}=0.15$. ABS-specific parameter $D_{\mathrm{m}}=1$ (thermal unit developmental model) was also used

outside this range, indicating the influence of factors such as human-induced mortality, mortality of non-adults, and stochasticity.

In addition to validation using real data and comparison with Leslie matrix model projections, we also conducted tests of the basic operation of the simulation such as the degree-hour accumulation for transition, age-specific mortality, and adult fecundity. These trials yielded the results that were expected, and thus are not presented here other than to say that they increased our confidence that the constituent functions of MED-FOES were working as expected.

Simulations of outbreaks

We executed 10,000 simulations of each outbreak listed in Table 2 using the parameters in Table 3. Parameter sets for each simulation were generated from the ranges shown in Table 3 by Latin Hypercube Sampling (Blower and 


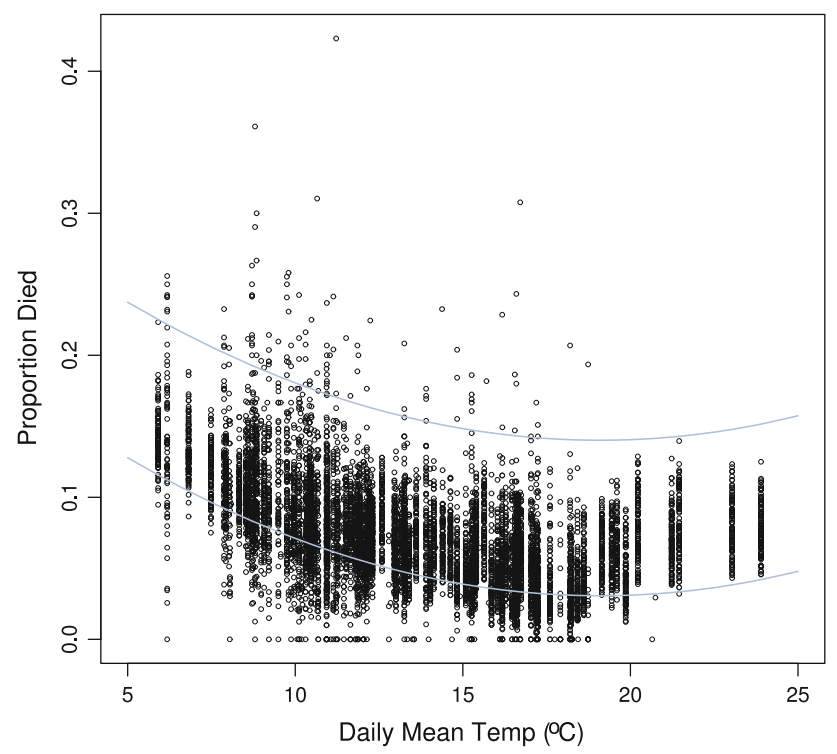

Fig. 3 The observed relationship between mean daily temperature and the proportion of agents in all stages that died on the same day for a randomly selected subset of 100 simulations of the Fallbrook outbreak. Data points for population sizes over 25 individuals only are included. The two gray lines represent the lower and upper boundaries of daily adult mortality set for all the simulations

Dowlatabadi 1994). Information on the times to extirpation $\left(t_{\mathrm{e}}\right)$ from these simulations is given in Table 4 , together with outbreak-specific parameters. In four out of seven cases the ABS showed extirpation in $95 \%$ of the simulations within 1 month of the quarantine lengths as prescribed by the CDFA, which are known to be effective through experience. However, in three cases (Escondido, Fallbrook, and Santa Monica) the ABS gave a much shorter length of time for extirpation of $95 \%$ of the simulations. The most extreme case, Escondido, can be explained by a cold snap in December 2009 (about 107 days after the initial find). The cold temperatures experienced in this area led to at least $4 \mathrm{~h}$ of temperatures below $0{ }^{\circ} \mathrm{C}$, which greatly increases mortality in the model and thus reduced time to the predicted extirpation. These low temperatures would have the opposite effect on quarantine length as determined by degree-day analysis, as the estimated development is significantly slowed or halted by such low temperatures.

We examined the results of the ABS of the Santa Monica outbreak further. Figure 4 shows the number of flies in a subset of the simulations performed. It is clear that population size stays within the range that has been suggested to be reasonable for Medfly finds in California (Cunningham and Couey 1986): a few hundred to thousands of adults, generally. It is also clear that some simulations have multiple generations, but not all, and the number of generations and the relative size of the population in each model run are highly variable. These results
Table 4 Outbreak-specific parameters and results from ABS on time to extirpation

\begin{tabular}{llrlllc}
\hline Outbreak & $N$ & \multicolumn{1}{c}{$t_{\mathrm{S}}$} & $t_{\mathrm{m}}$ & $t_{0.95}$ & $\mathrm{QL}$ & $t_{0.95}-\mathrm{QL}$ \\
\hline Santa Monica & $4167-6250$ & 2 & 160 & 231 & 297 & -66 \\
Fallbrook & $2760-4167$ & 12 & 143 & 206 & 273 & -67 \\
Spring Valley & $1375-2083$ & 5 & 136 & 200 & 204 & -4 \\
Imperial beach & $1375-2083$ & 4 & 123 & 172 & 145 & +27 \\
Mira Mesa & $1375-2083$ & 2 & 130 & 181 & 166 & +15 \\
Escondido & $1375-2083$ & 4 & 118 & 167 & 337 & -170 \\
Cajon & $2760-4167$ & 6 & 154 & 227 & 245 & -18 \\
\hline
\end{tabular}

$N$ initial population size, all stages, $t_{\mathrm{S}}$ time to human intervention (days), $t_{\mathrm{m}}$ mean time to extirpation from ABS (days), $t_{0.95}$ time for $95 \%$ of the ABS runs to show extirpation (days), $Q L$ length of quarantine determined by 3 generations of degree-day development (days)

indicate that the parameter ranges used for the ABS analysis of this outbreak produce a variety of possible population responses, including many realistic scenarios that may affect the actual outcome of the eradication program.

We conducted a sensitivity analysis of the ABS model for the Santa Monica outbreak using a partial rank correlation coefficient (PRCC) procedure (Kendall 1942). The PRCC test measures the strength of the association between our response variable $t_{\mathrm{e}}$ and each of the ABS input parameters while controlling for all other parameters in the model (see Table 5 for results).

\section{Discussion}

We have developed and implemented in the program MED-FOES an ABS designed to test quarantine lengths following a find of $C$. capitata in an area where it is not established. Our use of MED-FOES has yielded significant insights into population extirpation based on the actual outbreaks we simulated in this paper in two ways: (1) by comparison with the quarantine lengths set when the outbreaks occurred and (2) by analysis of the effect of simulation input parameters on its outcome. Below we discuss these two areas, place MED-FOES in the context of other insect ABS models, and conclude with expected future developments.

The predictive ability of MED-FOES for determining time to extirpation following Medfly outbreaks in California can be assessed against the length of quarantine imposed by CDFA, which is based on three generations of the deterministic degree-day development model (Table 4). The quarantine and countermeasures imposed for each of these seven outbreaks can be argued to have been successful in extirpating Medfly from the immediate area of the find (Dowell et al. 1999). This is thought to be the 
Fig. 4 Total number of flies in 500 simulations of the Santa Monica outbreak over time. Times are truncated to be less than 150 days. The red line represents the mean number of flies across simulations

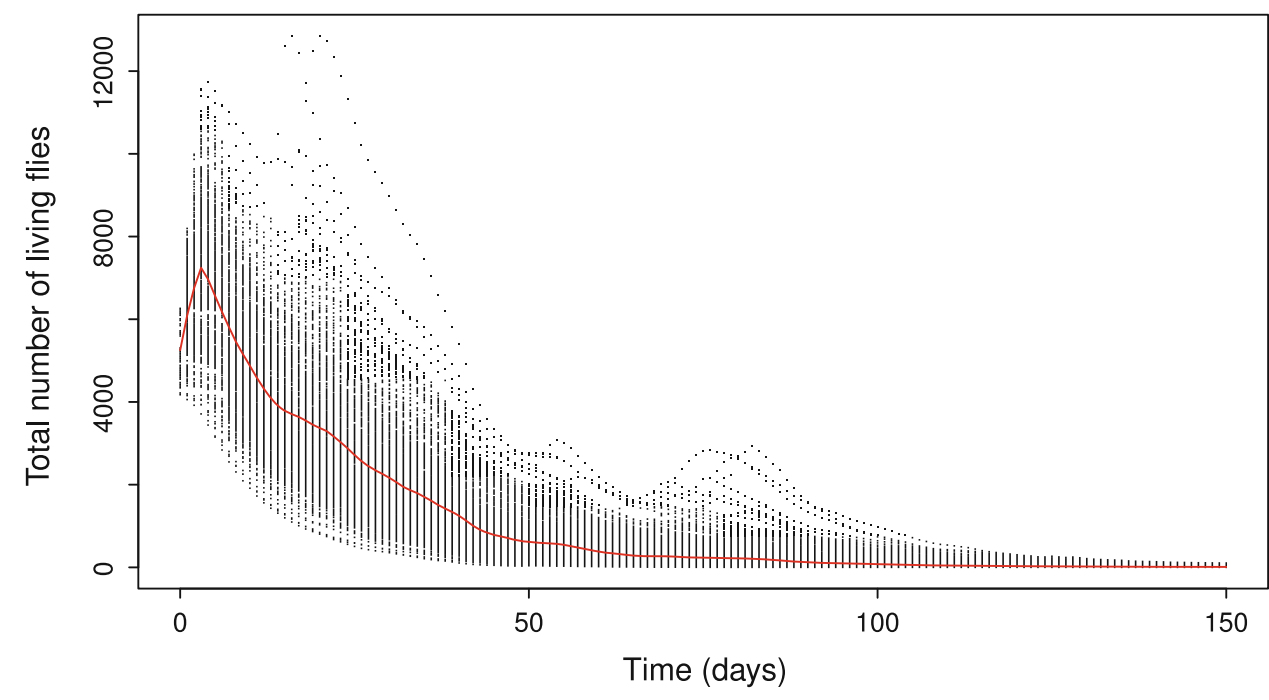

Table 5 Sensitivity of time to extirpation for individual simulations $\left(t_{\mathrm{e}}\right)$ to input parameters via partial rank correlation coefficient (PRCC) analysis

\begin{tabular}{lrrr}
\hline Input Parameter & Coefficient & Test statistic $(\rho)$ & $p$ \\
\hline$M_{\mathrm{p}}^{*}$ & -0.60 & -74.75 & $<0.0001$ \\
$M_{\mathrm{l}}^{*}$ & -0.45 & -50.81 & $<0.0001$ \\
$r_{\text {red }}$ & -0.42 & -45.97 & $<0.0001$ \\
$M_{\mathrm{a}}^{*}$ & -0.37 & -40.21 & $<0.0001$ \\
$T_{\text {min,p }}$ & 0.36 & 38.83 & $<0.0001$ \\
$r$ & 0.30 & 31.59 & $<0.0001$ \\
$M_{\mathrm{e}}^{*}$ & -0.24 & -25.23 & $<0.0001$ \\
$S$ & -0.15 & -15.33 & $<0.0001$ \\
$K_{\mathrm{a}}$ & -0.08 & -8.40 & $<0.0001$ \\
$N_{0}$ & 0.07 & 6.72 & $<0.0001$ \\
$K_{\mathrm{p}}$ & 0.06 & 5.89 & $<0.0001$ \\
$T_{\text {min,a }}$ & -0.06 & -5.58 & $<0.0001$ \\
$T_{\text {min,1 }}$ & -0.04 & 3.71 & 0.0002 \\
$K_{\mathrm{l}}$ & 0.02 & 1.85 & 0.0644 \\
$K_{\mathrm{e}}$ & 0.01 & 0.69 & 0.4909 \\
$T_{\text {min,e }}$ & -0.01 & -0.64 & 0.5234 \\
\hline
\end{tabular}

Entries in the table are ordered by the magnitude of the coefficients, which indicates the relative importance of each input parameter

case as detections were not made in the same local area after quarantine and treatment; however, they may not have totally eradicated the flies from the Southern California region. Indeed the outbreak data might be credibly interpreted to suggest that there was movement of flies from a single introduction in 2008 that led to the six subsequent finds through 2010. Though this hypothesis is not the topic here, it will influence future developments of the ABS, discussed below.

Our analysis with MED-FOES provides independent verification that the quarantine lengths set at the time of the outbreaks were appropriate for extirpation in the immediate area of the finds. This was the situation in most of the cases examined, but the margin of safety in the duration of the quarantine and control actions was not the same in all cases. Specifically, in the cases of Fallbrook, Santa Monica, and Escondido, the ABS suggests that the quarantines were much longer than necessary for a reasonable certainty of extirpation. In the Imperial Beach and Mira Mesa cases, the ABS called for a slightly longer time for $95 \%$ of the simulations to show extirpation, so the actual quarantine times implemented in these specific cases might not have yielded as high a certainty of extirpation as in the other outbreaks.

The ABS approach used in MED-FOES is a more realistic and comprehensive method of assessing and managing invasive Medfly populations following detection in an exclusion area when compared with the currently used degree-day approach. While the latter considers only the development of individuals, the ABS includes development together with demographic explicitness, mortality, and reproduction. Importantly, it also explicitly includes the countermeasures implemented by humans: SIT and bait sprays/increased trapping in the cases examined.

MED-FOES is more flexible than a degree-day calculation, and individual factors such as SIT may be excluded in cases where they do not apply, or other factors may be easily added into the simulation if other approaches are used for management. In addition, the ABS approach produced mean estimates of time to extirpation and also variances around these estimates, which the standard use of a degree-day calculation would not.

Systematic analysis of the behavior of the ABS can lead to novel insights into the changes in an invading population of $C$. capitata over time. The large difference between the quarantine lengths estimated by the degree-day approach 
versus the much shorter estimated time for extirpation from the ABS in the Escondido outbreak is an excellent example of how the ABS provides new insights and could perhaps save valuable resources for regulators and producers. At a regulatory level authorities can now consider cold snaps or any extreme condition as shortening the length of quarantine or even ending it, though they might have the opposite effect when using the currently standard developmental calculations of a degree-day model.

The sensitivity analysis presented in Table 5 shows that some parameters are of special importance to the simulation outcome aside from mortality for adults $\left(M_{\mathrm{a}}^{*}\right)$, which might be expected. In fact, the mortality at the optimum temperature for the larvae and pupae were more strongly related to $t_{\mathrm{e}}$ (time to extirpation). The relatively lower rank of $M_{\mathrm{a}}^{*}$ may be due to augmented human-induced mortality $(S)$ after intervention at $t_{\mathrm{S}}$. Sensitivity analysis of another agent-based model focusing on aphid population dynamics shows a similarly important role of mortality generally (Parry et al. 2006).

Reduction in reproductive agents through SIT $\left(r_{\text {red }}\right)$ was the third most important factor following pupal and larval base mortality. The top four parameters (mortality at optimum for pupae, larvae, and adults and $r_{\text {red }}$ ) play a large role in determining if there will be one, two, or more generations of Medfly after a detected outbreak. It is important to further measure these input parameters in live insects during the larval and pupal stages to refine future ABS investigations of Medfly incursions. It is interesting to note that the initial number of flies as determined by $N_{0}$ and the initial age distribution did not have a major effect on $t_{\mathrm{e}}$, indicating that the simulation is robust to errors in the estimate of the initial population size.

To our knowledge, agent-based approaches have not previously been applied to the question of quarantine lengths following insect invasions, though they have been used to address an array of other issues in insect biology, ranging from foraging networks and nest choice in ants (Jackson et al. 2004; Pratt et al. 2005) to landscape-level population studies (Arrignon et al. 2007; Babin-Fenske and Anand 2011; Perez and Dragicevic 2012) and disease vector dynamics (Almeida et al. 2010). The majority of the agent-based models on insects we have seen, including those cited above, are focused on adult behavior and how it scales up to population-level characteristics. The best recent models are often spatially explicit.

Though the current version of MED-FOES does not include adult behavior or explicit consideration of space, it does include a detailed developmental model, which is relatively uncommon in insect ABSs [but see Cormont et al. (2012) and Radchuk et al. (2013) for examples]. One of the important reasons that the detailed models of development are usually omitted is that a large number of developmental parameters measured in the lab or in nature are required for such a sub model to be included, and these are usually not available (Holcombe et al. 2012). This is the case even in ABS models of agricultural pests (CrespoPérez et al. 2011; Parry et al. 2006).

Including position and movement over a realistic landscape is an important goal for future versions of MEDFOES. Adding this factor will make it possible to address the important question of $C$. capitata establishment in California or other parts of the world. It will also be possible to test the hypothesis that a series of outbreaks may be linked by movement out of the quarantine area, leading to a find in a nearby location some time after extirpation in the original site has occurred.

MED-FOES is subject to issues common to all agentbased approaches. The most important is that to be realistic, the simulation will typically include many input parameters which need to be estimated from additional studies on real organisms. For $C$. capitata we are fortunate to have a large number of studies on development, mortality, and reproduction, but even for this species modeling movement at a landscape level will be difficult. A secondary but related problem is testing many combinations of these parameters across real-world environmental diversity, a process which can be computationally expensive. As a result of this added complexity, an ABS can often produce highly precise predictions that may not be particularly general or realistic (Levins 1966). The hourly simulation implemented in MED-FOES requires the best possible input data, specifically hourly temperature data. In some cases the nearest CIMIS weather station was over $20 \mathrm{~km}$ away, which might introduce inaccuracy in the simulation.

The ABS presented here might be generally considered a first draft of a framework for studying insect invasions. It can already be used to test if establishment of a given insect in a particular area is likely or unlikely, especially if sterility after human intervention is not assumed (this option is available in the current version of MED-FOES) and perhaps with extensions to consider temporal variation in habitat suitability (Arrignon et al. 2007). In the current paper we have used MED-FOES to gain insight into the time to extirpation of invasive Medfly in California focusing on the sufficiency of the length quarantine and intervention used by the state in seven outbreaks. Future development of this ABS is expected to include the implementation of males, adult behavior, and consideration of movement over the actual landscape of an outbreak and other spatially explicit elements (such as sterile flies and fruit stripping). These additional sub-models will allow for true interaction between the agents in the simulation, a common element of other ABS that can lead to emergent properties (Bedau 1997) from the system and allow a deeper exploration of insect invasions. 
Acknowledgments We thank R. Vargas, P. Follett, and E. Jang for sharing their expertise on Medfly and S. Geib and B. Hall for discussions on computational issues. We also thank S. Ellner for helpful advice on modeling decisions and R. Carruthers for improvements to the presentation. This work was funded by the United States Department of Agriculture-Agricultural Research Service (USDAARS). Opinions, findings, conclusions, or recommendations expressed in this publication are those of the authors and do not necessarily reflect the views of the USDA. USDA is an equal opportunity provider and employer.

Open Access This article is distributed under the terms of the Creative Commons Attribution License which permits any use, distribution, and reproduction in any medium, provided the original author(s) and the source are credited.

\section{References}

Arrignon F, Deconchat M, Sarthou J-P et al (2007) Modelling the overwintering strategy of a beneficial insect in a heterogeneous landscape using a multi-agent system. Ecol Model 205:423-436. doi:10.1016/j.ecolmodel.2007.03.006

Babin-Fenske J, Anand M (2011) Agent-based simulation of effects of stress on forest tent caterpillar (Malacosoma disstria Hübner) population dynamics. Ecol Model 222:2561-2569. doi:10.1016/ j.ecolmodel.2011.04.014

Back EA, Pemberton CE (1918) The Mediterranean fruit fly in Hawaii. U.S. Dept. of Agriculture, Washington DC

Bedau MA (1997) Weak emergence. Nous 31:375-399

Blower SM, Dowlatabadi H (1994) Sensitivity and uncertainty analysis of complex models of disease transmission: an HIV model, as an example. Int Stat Rev 2:229-243

Bonabeau E (2002) Agent-based modeling: methods and techniques for simulating human systems. Proc Natl Acad Sci USA 99:7280-7287. doi:10.1073/pnas.082080899

Bonizzoni M, Zheng L, Guglielmino C et al (2001) Microsatellite analysis of medfly bioinfestations in California. Mol Ecol 10:2515-2524

Campbell GS, Norman JM (1997) An introduction to environmental biophysics, 2nd edn. Springer, New York

Campbell A, Frazer BD, Gilbert N et al (1974) Temperature requirements of some aphids and their parasites. J Appl Ecol 11:431-438. doi:10.2307/2402197

Carey JR (1982) Demography and population dynamics of the Mediterranean fruit fly. Ecol Model 16:125-150. doi:10.1016/ 0304-3800(82)90005-9

Carey JR (1991) Establishment of the Mediterranean fruit fly in California. Science 253:1369

Carey JR (1992) The Mediterranean fruit fly in California: taking stock. Calif Agric 46:12-17

Carey JR (1996) The future of the Mediterranean fruit fly Ceratitis capitata invasion of California: a predictive framework. Biol Conserv 78:35-50. doi:10.1016/0006-3207(96)00016-X

Carey JR (2011) Biodemography of the Mediterranean fruit fly: aging, longevity and adaptation in the wild. Exp Gerontol 46:404-411. doi:10.1016/j.exger.2010.09.009

CDFA (2012) California Dept of Food and Agriculture, Medfly Quarantine Information. Sacramento CA

Christenson L, Foote RH (1960) Biology of fruit flies. Annu Rev Entomol 5:171-192

Cormont A, Jochem R, Malinowska A et al (2012) Can phenological shifts compensate for adverse effects of climate change on butterfly metapopulation viability? Ecol Model 227:72
Crespo-Pérez V, Rebaudo F, Silvain JF, Dangles O (2011) Modeling invasive species spread in complex landscapes: the case of potato moth in Ecuador. Landsc Ecol 26(10):1447-1461

Cunningham RT, Couey HM (1986) Mediterranean fruit fly (Diptera: Tephritidae): Distance/response curves to trimedlure to measure trapping efficiency. Environ Entomol 15:71-74

Davies N, Villablanca FX, Roderick GK (1999) Bioinvasions of the Medfly Ceratitis capitata: source estimation using DNA sequences at multiple intron loci. Genetics 153:351-360

de Almeida SJ, Martins Ferreira RP, Eiras ÁE et al (2010) Multiagent modeling and simulation of an Aedes aegypti mosquito population. Environ Model Softw 25:1490-1507. doi:10.1016/ j.envsoft.2010.04.021

DeAngelis D, Rose K, Huston M (1994) Individual-oriented approaches to modeling ecological populations and communities. In: Levin $\mathrm{S}$ (ed) Frontiers in mathematical biology. Springer, New York, pp 390-410

Diamantidis AD, Papadopoulos NT, Nakas CT et al (2009) Life history evolution in a globally invading tephritid: patterns of survival and reproduction in Medflies from six world regions. Biol J Linn Soc 97:106-117. doi:10.1111/j.1095-8312.2009.01178.x

Dowell R, Siddiqui I, Meyer F et al (1999) Early results suggest sterile flies may protect S. California from Medfly. Calif Agric 53:28-32

Dowell RV, Siddiqu I, Meyer F et al (2000) Mediterranean fruit fly preventative release programme in Southern California. Areawide control of fruit flies and other insect pests. In: Joint proceedings of the international conference on area-wide control of insect pests, 28 May to 2 June 1998 and the Fifth International Symposium on Fruit Flies of Economic Importance, Penang, Malaysia, 1-5 June 1998, pp 369-375

Duyck P f, Quilici S (2002) Survival and development of different life stages of three Ceratitis spp. (Diptera: Tephritidae) reared at five constant temperatures. Bull Entomol Res 92:461-469. doi:10.1079/BER2002188

El Keroumi A, Naamani K, Dahbi A et al (2010) Effect of ant predation and abiotic factors on the mortality of Medfly larvae, Ceratitis capitata, in the Argan forest of Western Morocco. Biocontrol Sci Technol 20:751-762. doi:10.1080/09583151 003734651

Fletcher BS (1989) Temperature-development rate relationships of the immature stages and adults of tephritid fruit flies. In: Robinson AS, Hooper G (eds) Fruit flies: their biology, natural enemies, and control. Elsevier, Amsterdam, pp 273-289

Fry J, Taylor CE, Devgan U (1989) An expert system for mosquito control in Orange County California. Bull Soc Vector Ecol $14: 237-246$

Gasperi G, Bonizzoni M, Gomulski L et al (2002) Genetic differentiation, gene flow and the origin of infestations of the Medfly, Ceratitis capitata. Genetica 116:125-135

Gilbert A, Bingham R, Nicolas M, Clark R (2010) Insect trapping guide, 12th edn. CDFA, Sacramento

Grimm V (1999) Ten years of individual-based modelling in ecology: what have we learned and what could we learn in the future? Ecol Model 115:129-148

Grimm V, Berger U, Bastiansen F et al (2006) A standard protocol for describing individual-based and agent-based models. Ecol Model 198:115-126. doi:10.1016/j.ecolmodel.2006.04.023

Grimm V, Berger U, DeAngelis DL et al (2010) The ODD protocol: a review and first update. Ecol Model 221:2760-2768

Grout TG, Stoltz KC (2007) Developmental rates at constant temperatures of three economically important Ceratitis spp. (Diptera: Tephritidae) from Southern Africa. Environ Entomol 36:1310-1317. doi:10.1603/0046-225X

Grout TG, Daneel JH, Ware AB, Beck RR (2011) A comparison of monitoring systems used for Ceratitis species (Diptera: 
Tephritidae) in South Africa. Crop Prot 30:617-622. doi:10.1016/j.cropro.2011.01.005

Gutierrez AP, Ponti L (2011) Assessing the invasive potential of the Mediterranean fruit fly in California and Italy. Biol Invasions 13:2661-2676. doi:10.1007/s10530-011-9937-6

Hales D, Rouchier J, Edmonds B (2003) Model-to-model analysis. J Artif Soc Soc Simul 6:5

Harris EJ, Carey JR, Krainacker DA, Lee CYL (1991) Life history of Ceratitis capitata (Diptera: Tephritidae) reared from mock orange in Hawaii. Environ Entomol 20:1048-1052

Haymer DS, He M, McInnis DO (1997) Genetic marker analysis of spatial and temporal relationships among existing populations and new infestations of the Mediterranean fruit fly (Ceratitis capitata). Heredity 79:302-309

Holcombe M, Adra S, Bicak M et al (2012) Modelling complex biological systems using an agent-based approach. Integr Biol 4:53. doi: $10.1039 / \mathrm{c} 1 \mathrm{ib} 00042 \mathrm{j}$

Huston M, DeAngelis D, Post W (1988) New computer models unify ecological theory. BioScience 38:682-691

Jackson DE, Holcombe M, Ratnieks FL (2004) Trail geometry gives polarity to ant foraging networks. Nature 432:907-909

Kaspi R, Mossinson S, Drezner T et al (2002) Effects of larval diet on development rates and reproductive maturation of male and female Mediterranean fruit flies. Physiol Entomol 27:29-38. doi:10.1046/j.1365-3032.2001.00264.x

Katsoyannos B, Heath R, Papadopoulos N et al (1999) Field evaluation of Mediterranean fruit fly (Diptera: Tephritidae) female selective attractants for use in monitoring programs. J Econ Entomol 92:583-589

Kendall MG (1942) Partial rank correlation. Biometrika 32:277-283

Knipling EF (1979) The basic principles of insect population suppression and management. U.S. Dept. of Agriculture, Washington DC

Lance DR, Gates DB (1994) Sensitivity of detection trapping systems for Mediterranean fruit flies (Diptera: Tephritidae) in Southern California. J Econ Entomol 87:1377-1383

Leslie PH (1945) On the use of matrices in certain population mathematics. Biometrika 33:183-212

Levins R (1966) The strategy of model building in population biology. Am Sci 54:421-431

Lewis E (1942) On the generation and growth of a population. Sankhya 6:93-96

Liquido NJ, Cunningham RT, Nakagawa S (1990) Host plants of Mediterranean fruit fly (Diptera: Tephritidae) on the island of Hawaii (1949-1985 survey). J Econ Entomol 83:1863-1878

Macal CM, North MJ (2010) Tutorial on agent-based modelling and simulation. J Simul 4:151-162

Messenger PS (1959) Bioclimatic Studies with Insects. Annu Rev Entomol 4:183-206. doi:10.1146/annurev.en.04.010159.001151

Messenger PS, Flitters NE (1958) Effect of constant temperature environments on the egg stage of three species of Hawaiian fruit flies. Ann Entomol Soc Am 51:109-119

Midgarden D, Ovalle O, Epsky ND et al (2004) Capture of Mediterranean fruit flies (Diptera: Tephritidae) in dry traps baited with a food-based attractant and Jackson traps baited with trimedlure during sterile male release in Guatemala. J Econ Entomol 97:2137-2143

Papachristos DP, Papadopoulos NT, Nanos GD (2008) Survival and development of immature stages of the Mediterranean fruit fly (Diptera: Tephritidae) in citrus fruit. $\mathrm{J}$ Econ Entomol 101:866-872. doi:10.1603/0022-0493
Papadopoulos NT, Katsoyannos BI, Carey JR (2002) Demographic parameters of the Mediterranean fruit fly (Diptera: Tephritidae) reared in apples. Ann Entomol Soc Am 95:564-569. doi:10.1603/0013-8746

Parry HR, Evans AJ, Morgan D (2006) Aphid population response to agricultural landscape change: a spatially explicit, individualbased model. Ecol Model 199:451-463. doi:10.1016/ j.ecolmodel.2006.01.006

Perez L, Dragicevic S (2012) Landscape-level simulation of forest insect disturbance: coupling swarm intelligent agents with GISbased cellular automata model. Ecol Model 231:53-64. doi:10.1016/j.ecolmodel.2012.01.020

Pratt SC, Sumpter DJT, Mallon EB, Franks NR (2005) An agentbased model of collective nest choice by the ant Temnothorax albipennis. Anim Behav 70:1023-1036. doi:10.1016/ j.anbehav.2005.01.022

Radchuk V, Johst K, Groeneveld J et al (2013) Behind the scenes of population viability modeling: predicting butterfly metapopulation dynamics under climate change. Ecol Model 259:62-73

Railsback SF, Grimm V (2012) Agent-based and individual-based modeling: a practical introduction. Princeton University Press, Princeton

Rivnay E (1950) The Mediterranean fruit fly in Israel. Bull Entomol Res 41:321-341

Rossler Y (1975) Reproductive differences between laboratory-reared and field-collected populations of the Mediterranean fruit fly, Ceratitis capitata. Ann Entomol Soc Am 68:987-991

Sawyer AJ, Feng Z, Hoy CW et al (1987) Instructional simulation: sterile insect release method with spatial and random effects. Bull Entomol Soc Am 33:182-190

Shoukry A, Hafez M (1979) Studies on the biology of the Mediterranean fruit fly Ceratitis capitata. Entomol Exp Appl 26:33-39. doi:10.1111/j.1570-7458.1979.tb02894.x

Siebert J, Cooper T (1995) If Medfly infestation triggered a trade ban: embargo on California produce would cause revenue, job loss. Calif Agric 49:7-12

Simberloff D, Schmitz DC, Brown TC (1997) Strangers in paradise: impact and management of nonindigenous species in Florida. Island Press, Washington DC

Steiner L (1967) Methods of estimating the size of populations of sterile pest Tephritidae in release programs. In: Proceedings of a panel organized by the joint FAO/IAEA Division of Atomic Energy in Food and Agriculture, Vienna. p 63

Steiner L (1969) A method of estimating the size of native populations of Oriental, Melon, and Mediterranean fruit flies to establish the over flooding ratios required for sterile-male releases. J Econ Entomol 62:4-7

Vargas RI, Walsh WA, Jang EB et al (1996) Survival and development of immature stages of four Hawaiian fruit flies (Diptera: Tephritidae) reared at five constant temperatures. Ann Entomol Soc Am 89:64-69

Vargas RI, Walsh WA, Kanehisa D et al (1997) Demography of four Hawaiian fruit flies (Diptera: Tephritidae) reared at five constant temperatures. Ann Entomol Soc Am 90:162-168

Vargas RI, Walsh WA, Kanehisa D et al (2000) Comparative demography of three Hawaiian fruit flies (Diptera: Tephritidae) at alternating temperatures. Ann Entomol Soc Am 93:75-81. doi:10.1603/0013-8746

Vinatier F, Tixier P, Duyck PF, Lescourret F (2011) Factors and mechanisms explaining spatial heterogeneity: a review of methods for insect populations. Method Ecol Evol 2:11-22 